models of experimental fibrosis. Thus, the Hh pathway could be an interesting novel target for the treatment of SSc.

\section{A66 ACTIVATION OF HEDGEHOG SIGNALLING PLAYS A CRUCIAL ROLE IN THE PATHOGENESIS OF SYSTEMIC SCLEROSIS}

Angelika Horn, ${ }^{1}$ Clara Dees, ${ }^{1}$ Alfiya Akhmetshina, ${ }^{1}$ Katrin Palumbo, ${ }^{1}$ Nicole Busch, ${ }^{1}$ Oliver Distler, ${ }^{2}$ Georg Schett, ${ }^{1}$ Jörg H W Distler ${ }^{1}$ Department of Internal Medicine, University of Erlangen, Erlangen, Germany; ${ }^{2} \mathrm{Ctr}$ Exp Rheum and Zurich Ctr Integr Hum Physiol, Univ Hosp, Zurich, Switzerland; ${ }^{3}$ Univ Erlangen, Germany

\subsection{6/ard.2010.129601f}

Purpose The Hedgehog (Hh) pathway plays important roles in embryonic tissue development, in the pathogenesis of various tumours and in hepatic fibrosis. The ligand Sonic Hedgehog (Shh) binds to the transmembrane receptor Patched, which releases the membrane protein Smoothened (Smo) from its repression. As a result, Smo activates the transcription factor GLI-Kruppel family member (Gli2) for further activation of several target genes.

Methods The expression of Shh, Smo and Gli2 in human skin sections was analysed by immunohistochemistry. Hh signalling was activated by recombinant Shh in vitro or adenoviral expression of Shh in vivo. Two different animal models of SSc, bleomycin-induced dermal fibrosis and tight-skin (tsk-1) mice were used to assess the role of Hh signalling in experimental fibrosis and to evaluate the anti-fibrotic potential of a specific Hh pathway inhibitor (LDE223) and of siRNA against Smo.

Results The expression of Shh and its downstream target Gli2 was significantly elevated in skin sections of SSc patients compared to healthy controls. Similarly, Hh signalling was also activated in different models of experimental fibrosis. Stimulation of healthy dermal fibroblasts with recombinant Shh induced a SSc-like phenotype and stimulated the release of collagen dose-dependently by up to $4.4 \pm 0.2$-fold. In addition, stimulation of fibroblasts with Shh induced the differentiation of myofibroblasts. Moreover, adenoviral overexpression of Shh-induced dermal fibrosis in mice with dermal thickening, increased accumulation of collagen and myofibroblast differentiation. In the mouse model of bleomycin-induced fibrosis, dermal thickening was reduced by $72 \pm 2 \%$ upon treatment with LDE223. Consistent with the reduced dermal thickness, LDE223-treated mice showed significantly decreased myofibroblast counts and the amount of collagen in the skin was reduced by up to $67 \pm 17 \%$. Similar results were obtained for Smo siRNA in the mouse model of bleomycin-induced skin fibrosis. Treatment with LDE223 also effectively prevented fibrosis in tsk-1 mice with reduction of hypodermal thickening by up to $78 \pm 5 \%$.

Conclusion The Hh pathway is activated in experimental models of SSc and in SSc patients. We demonstrate that activation of $\mathrm{Hh}$ activates cultured fibroblasts in vitro and induces fibrosis in vivo. Inhibition of Hh signalling by selective inhibition of Smo exerts potent anti-fibrotic effects in different 